Supporting Information for

\title{
Synthesis, Structural Characterization, and Catalytic Performance of Dititanium-substituted $\gamma$-Keggin Silicotungstate
}

\author{
Yuya Goto, ${ }^{\dagger}$ Keigo Kamata, ${ }^{\dagger}$ Kazuya Yamaguchi, ${ }^{\dagger, \dagger}$ Kazuhiro Uehara, ${ }^{\ddagger}$ \\ Shiro Hikichi, ${ }^{\dagger, \star}$ and Noritaka Mizuno $*, \dagger, \star$
}

$\dagger$ Department of Applied Chemistry, School of Engineering, The University of Tokyo, 7-3-1 Hongo, Bunkyo-ku, Tokyo 113-8656, Japan.

¥ Core Research for Evolutional Science and Technology (CREST), Japan Science and Technology Agency (JST), 4-1-8 Honcho, Kawaguchi, Saitama, 332-0012, Japan. tmizuno@mail.ecc.u-tokyo.ac.jp

\section{S-1 Details of X-ray crystallography.}

Single crystal X-ray measurements were made on Rigaku AFC-10 Saturn 70 CCD area detector equipped with Mo $\mathrm{K} \alpha(\lambda=0.71070 \AA)$. All data collections were carried out at $-180{ }^{\circ} \mathrm{C}$ using CrystalClear ${ }^{1}$ and indexing, integration, and absorption correction were performed with HKL 2000 software for Linux. ${ }^{2}$

Details of crystallography were as follows: a molecular structure of TBA-1 was solved by a combination of SHELXS-97 (direct method) and SHELXH-97 (Fourier and least squares refinement) ${ }^{3}$ linked to Win-GX for Windows software. ${ }^{4}$ The unit cell contains two crystallographic independent molecules. The atom labels were added to A 
and B (for Molecule A) or B and C (for Molecule B) as a suffix for clarity. Titanium and tungsten atoms were refined anisotropically and all the atoms except for metal atoms were refined isotropically. Total 16 molecules of tetra( $n$-butyl)ammonium cation (TBA) were observed, however, the part of the TBA moieties could not be observed due to highly disordered alkyl groups.

Molecular structures of CEK•1 and $\mathbf{2}$ were solved by a combination of DIRDIF PATTY (patterson method) and DIRDIF (Fourier synthesis) $)^{5,6}$ linked to CrystalStructure for Windows software. ${ }^{7}$ In the final full-matrix least squares refinement process, the atoms except for [K(18-crown-6)] moieties were refined anisotropically and several [K(18-crown-6)] fragments refined isotropically, or located in calculated positions and not refined [for CEK·1: (C501-C512, O501-O506), (C601-C612, O601-O606) and (C701-C712, O701-O706); for 2: (C201-C212, O201-O206), (C301-C312, O301-O306), (C412-C412, O401-O402), and (C501-C512, O501-O506)]. Occupancies of disordered atoms in CEK·1 [(C1, C2), (O201, O211), $(\mathrm{K} 1, \mathrm{~K} 11)$ and $(\mathrm{K} 5, \mathrm{~K} 55)]$ were calculated to be $[(0.5,0.5),(0.5018,0.4982),(0.5433$, $0.4567)$ and $(0.6289,0.3711)]$, respectively. In addition, [K(18-crown-6)] moiety containing $\mathrm{K} 4$ in $\mathbf{2}$ possesses the crystallographic mirror plane. Three of four fragments in unit cell ideally exist, so that occupancy of this fragment is determined to be 0.75 . In 2, the occupancies of the carbon atoms of the OMe ligands ( $\mathrm{C} 1$ and $\mathrm{C} 2)$ are refined as 0.5 due to the crystallographic site disordering. 


\section{References}

1. (a) CrystalClear 1.3.5 SP2: Rigaku and Rigaku/MSC. (b) Pflugrath J.W. Acta Crystallogr. 1999, D55, 1718-1725.

2. Otwinowski, Z.; Minor, W. Processing of X-ray Diffraction Data Collected in Oscillation Mode, Methods in Enzimology, Vol 276 (Eds: Carter, C. W., Jr.; Sweet, R. M.); Macromolecular Crystallography, part A, Academic Press, New York, 1997, pp. $307-326$

3. Sheldrick, G. M. SHELX97, Programs for Crystal Structure Analysis (Release 97 - 2), University of Göttingen, Göttingen (Germany), 1997.

4. Farrugia, L. J. J. Appl. Cryst. 1999, 32, 837-838.

5. Beurskens, P. T.; Admiraal, G.; Beurskens, G.; Bosman, W. P.; Garcia-Granda, S.; Gould, R. O.; Smits, J. M. M.; Smykalla, C. The DIRDIF program system, Technical Report of the Crystallography Laboratory, University of Nijmegen, Netherlands, 1992.

6. Beurskens, P. T.; Admiraal, G.; Beurskens, G.; Bosman, W. P.; de Gelder, R.; Israel, R.; Smits, J. M. M. The DIRDIF-99 program system, Technical Report of the Crystallography Laboratory, University of Nijmegen, Netherlands, 1999.

7. CrystalStructure 3.7.0: Crystal Structure Analysis Package, Rigaku and Rigaku/MSC. 


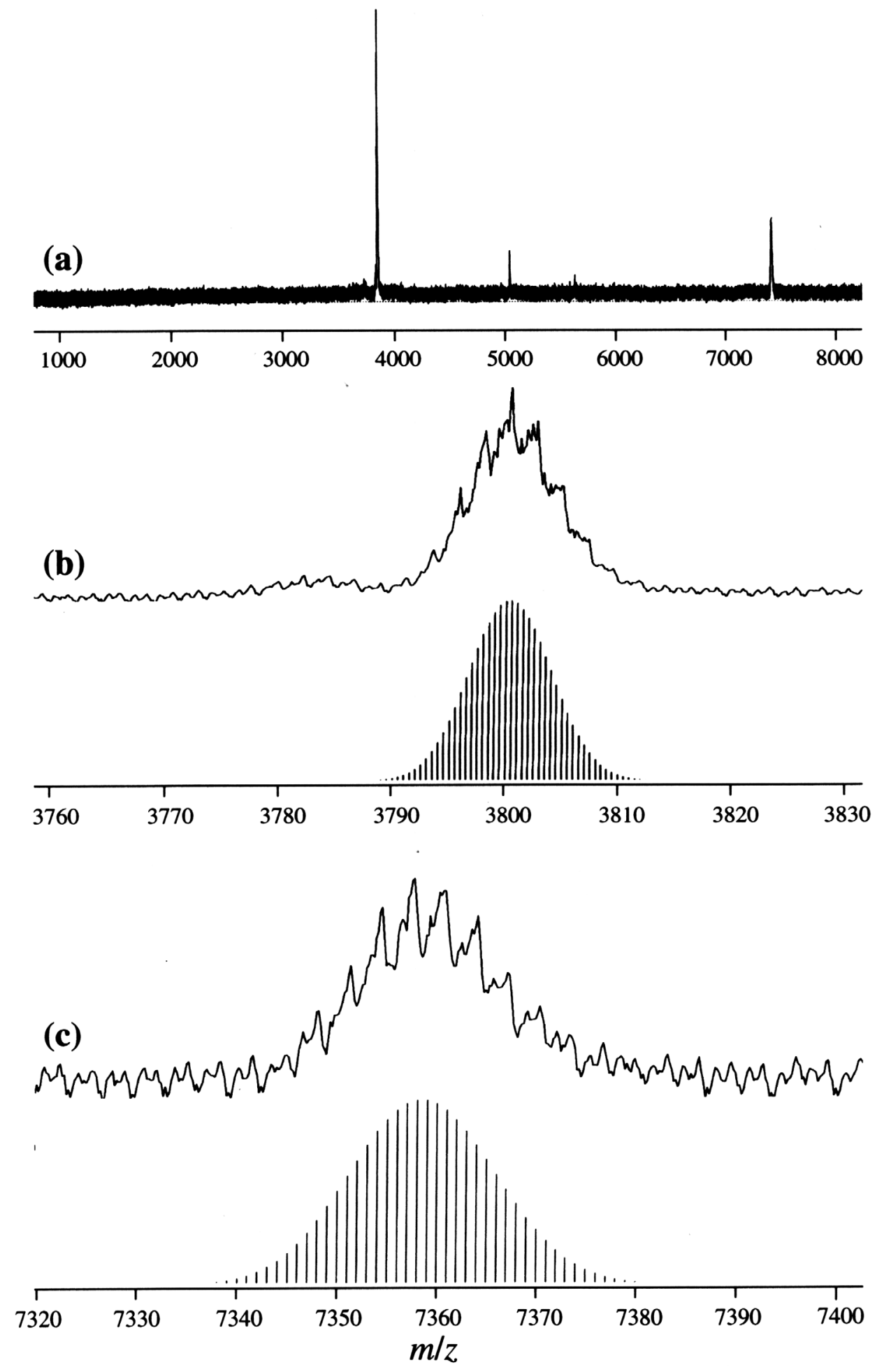

Figure S1. Positive ion CSI-MS spectra of TBA-1 in MeCN ((a) $m / z$ 1000-8000, (b) $m / z$ 3760-3830, and (c) $m / z$ 7320-7400). The lines in (b) and (c) are the calculated pattern of $\left\{\left(\left(\mathrm{C}_{4} \mathrm{H}_{9}\right)_{4} \mathrm{~N}\right)_{10}\left[\mathrm{H}_{4} \mathrm{Si}_{2} \mathrm{Ti}_{4} \mathrm{~W}_{20} \mathrm{O}_{78}\right]\right\}^{2+}$ and $\left\{\left(\left(\mathrm{C}_{4} \mathrm{H}_{9}\right)_{4} \mathrm{~N}\right)_{9}\left[\mathrm{H}_{4} \mathrm{Si}_{2} \mathrm{Ti}_{4} \mathrm{~W}_{20} \mathrm{O}_{78}\right]\right\}^{+}$, respectively . 
S-5 


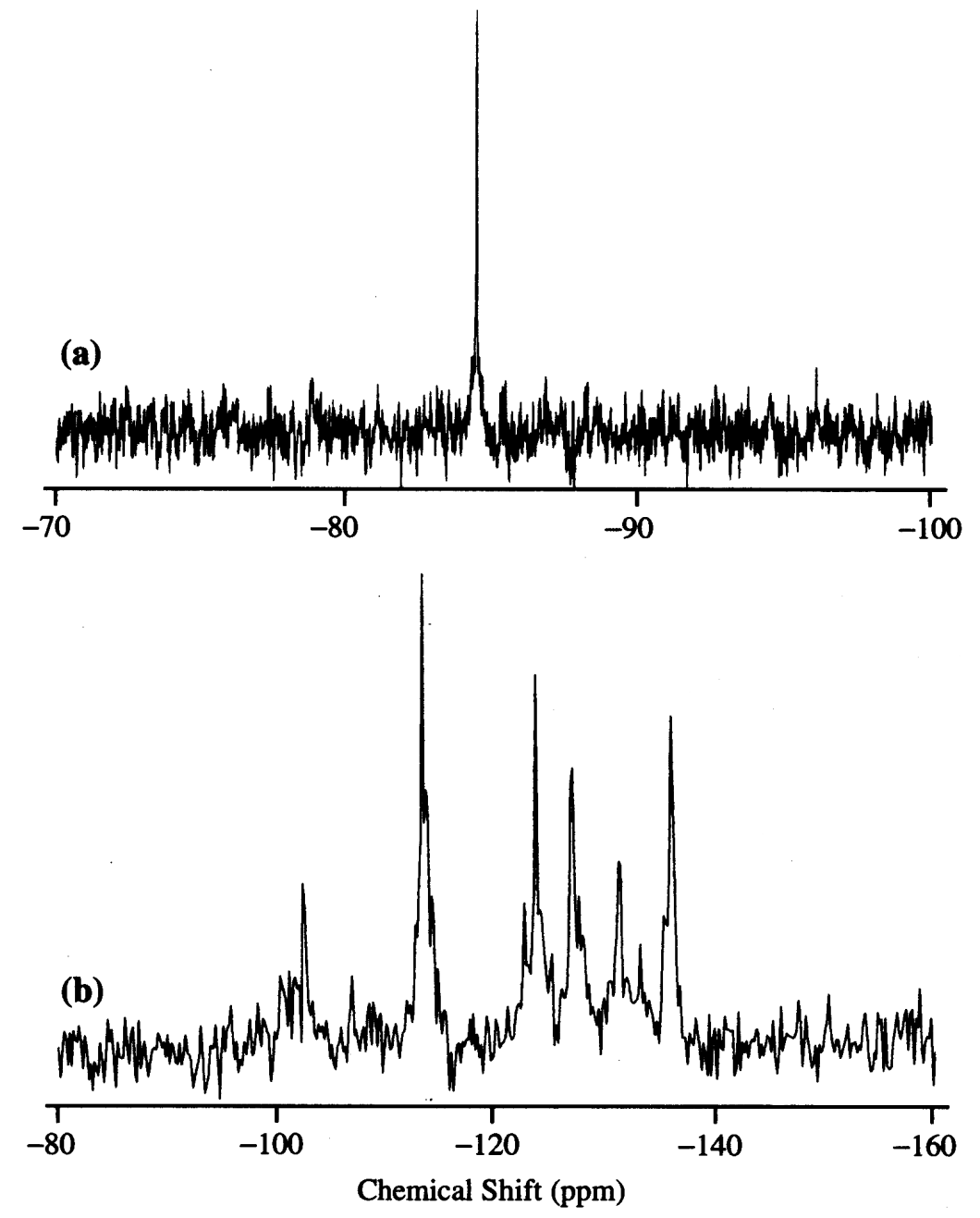

Figure S2. ${ }^{29} \mathrm{Si}$ NMR (a) and ${ }^{183} \mathrm{~W}$ NMR (b) spectra of TBA salt of 2. 


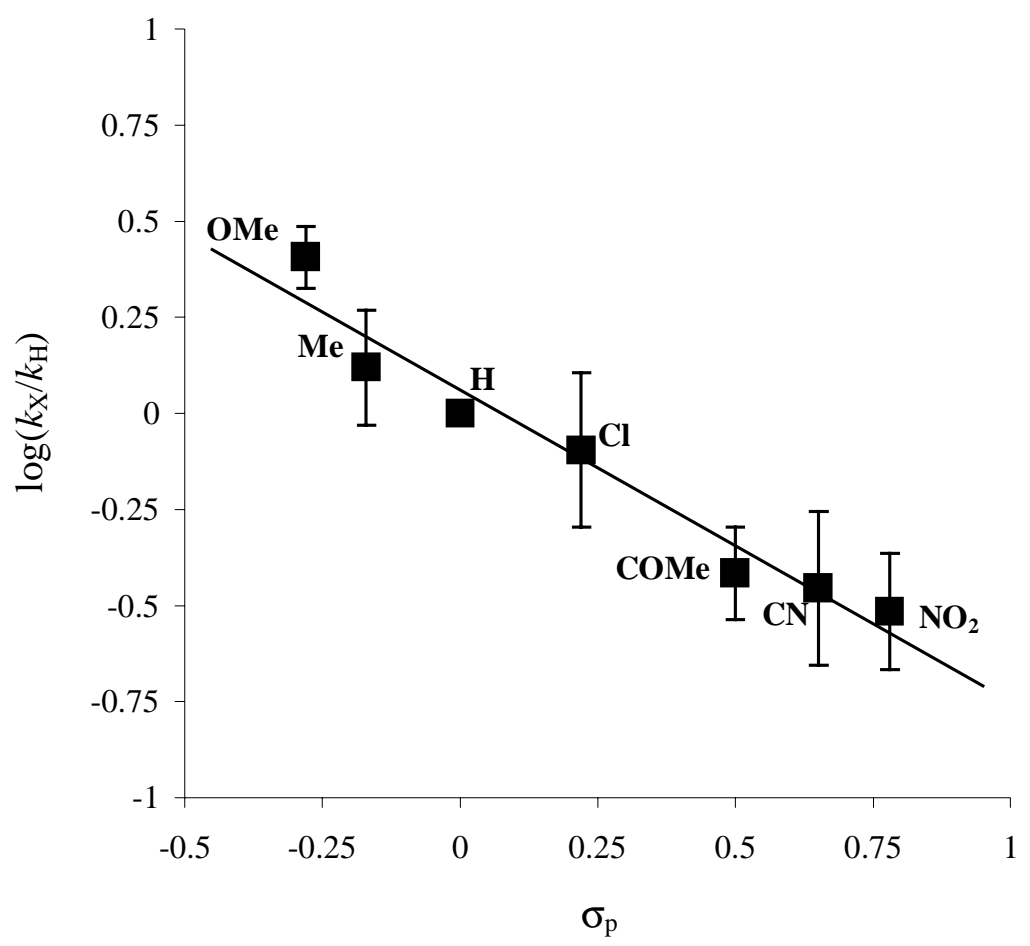

Figure S3. Hammett plots $\left(\log \left(k_{\mathrm{X}} / k_{\mathrm{H}}\right)\right.$ vs. $\sigma_{\mathrm{p}}$ plots $)$ for competitive oxidation of thioanisol and $p$-substituted thioanisols. Reaction conditions: Thioanisol $(0.5 \mathrm{mmol}), p$-substituted thioanisol (0.5 mmol), TBA•1 (2 $\mu \mathrm{mol}), \mathrm{H}_{2} \mathrm{O}_{2}$ (30\% aq., $\left.0.2 \mathrm{mmol}\right), \mathrm{CD}_{3} \mathrm{CN}(1 \mathrm{~mL}), 293 \mathrm{~K}$. Slope $=-0.81\left(r^{2}=0.95\right)$. 


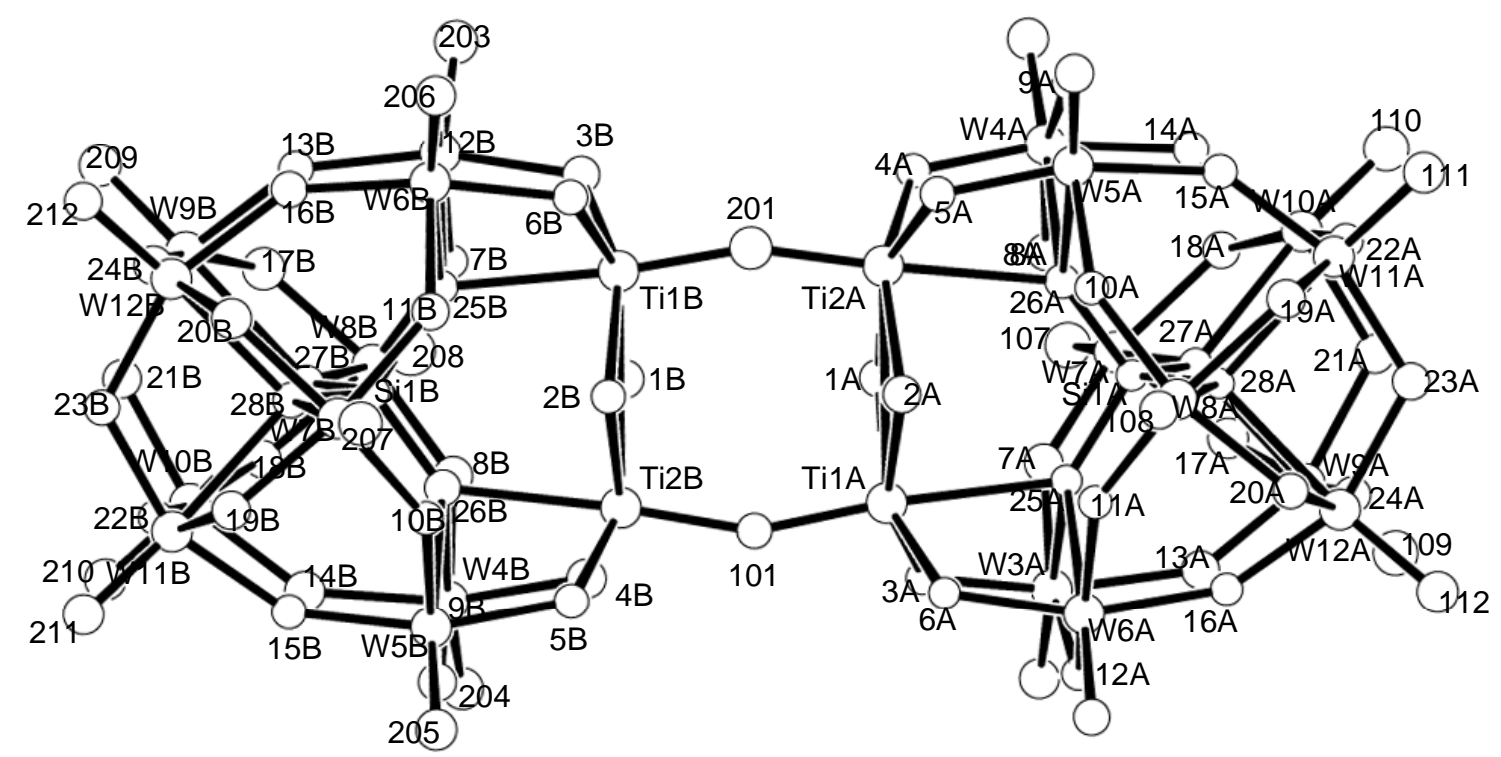

Figure S4. Numbering scheme of Molecule A in TBA $\mathbf{1}$. Atoms only with the number label represent oxygen atoms.

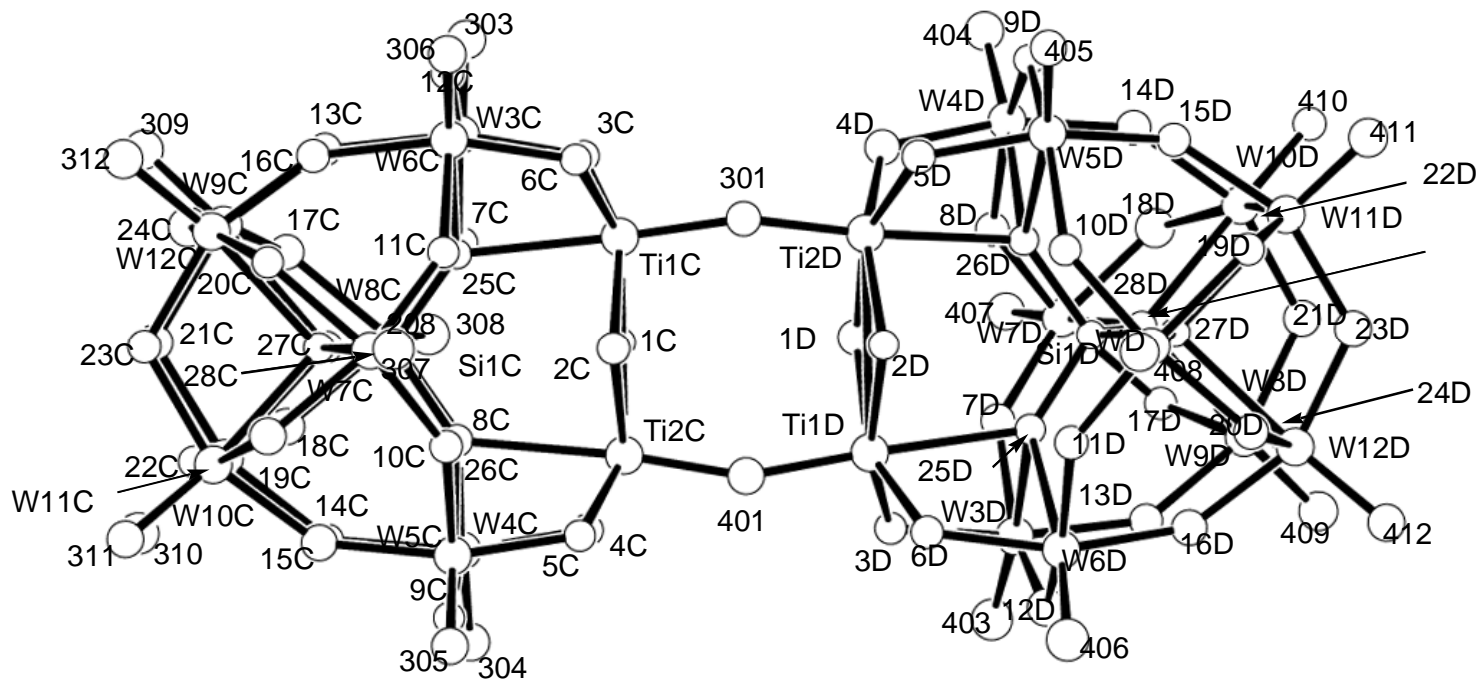

Figure S5. Numbering scheme of Molecule B in TBA $\mathbf{1}$. The atoms only with the number label represent oxygen atoms. 


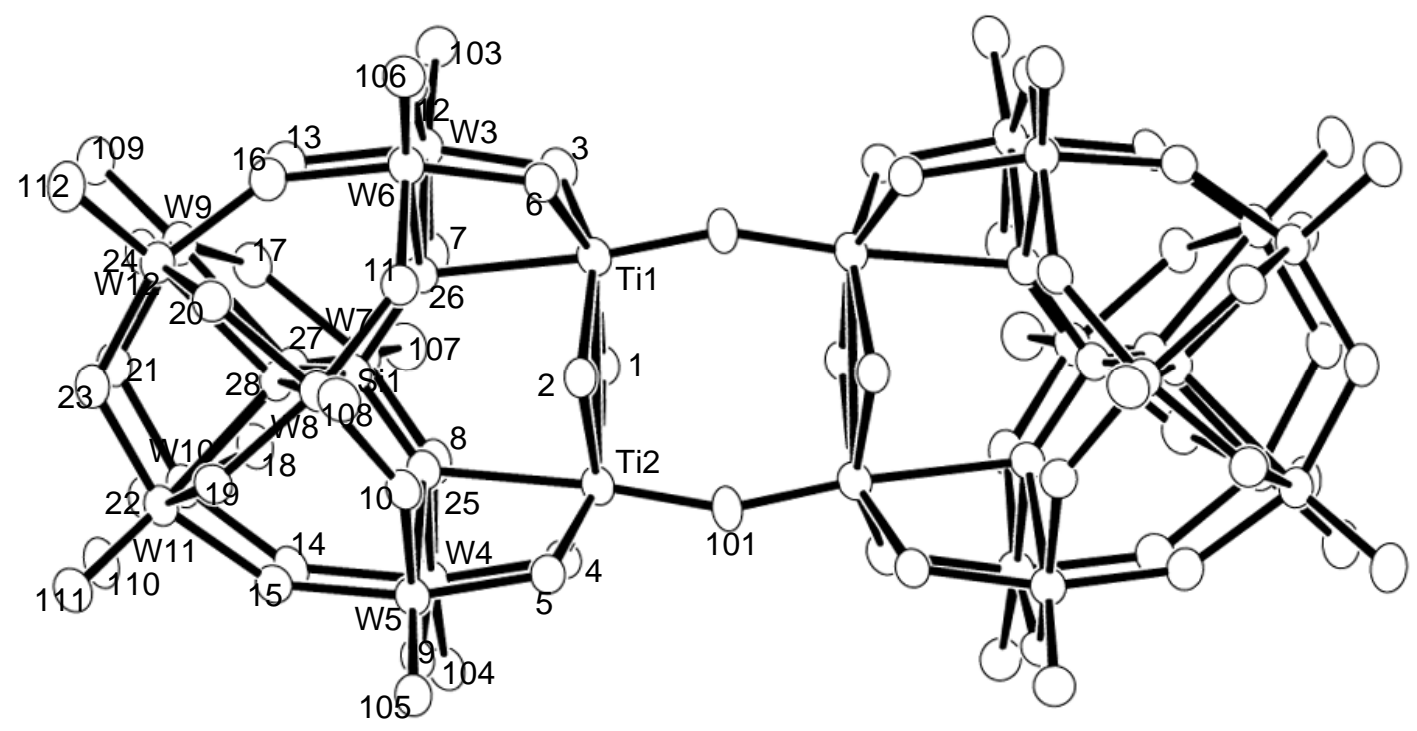

Figure S6. Numbering scheme of CEK·1. Atoms only with the number label represent oxygen atoms.

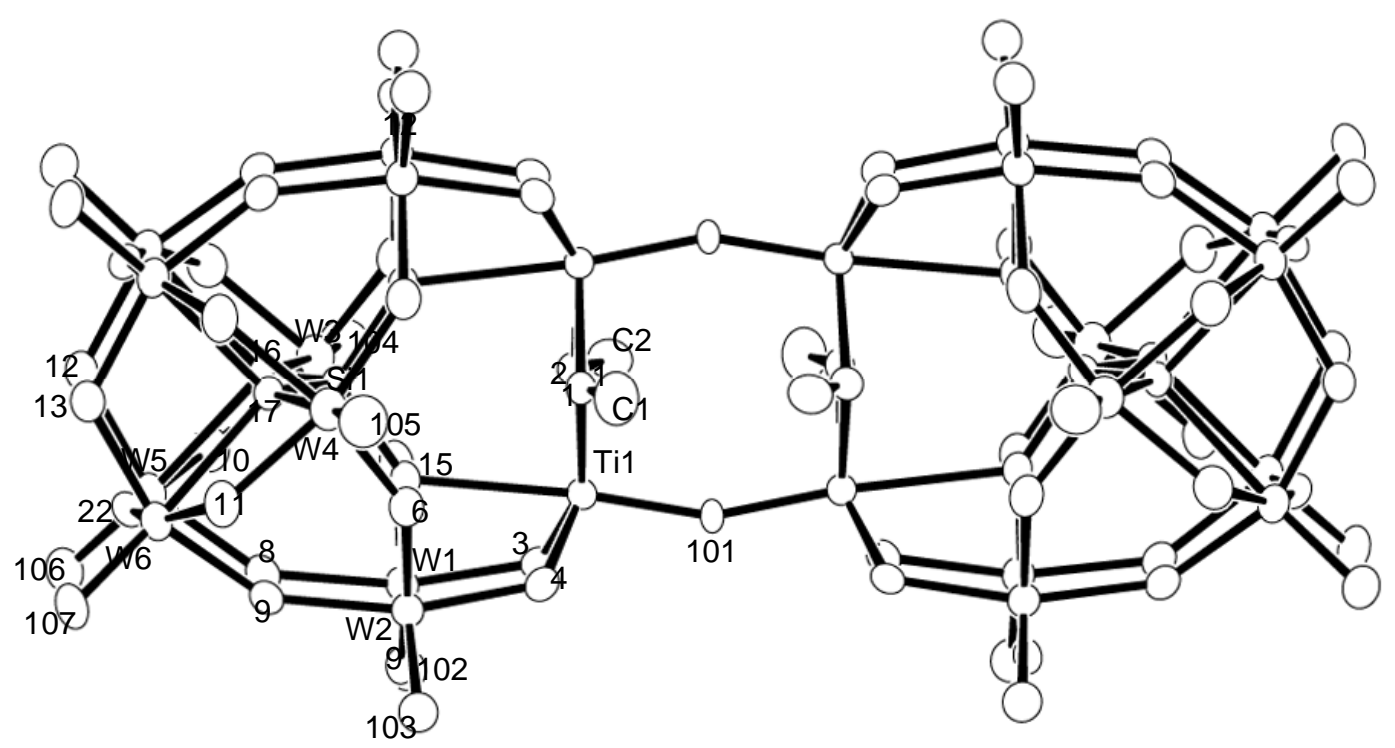

Figure S7. Numbering scheme of 2. Atoms only with the number label represent oxygen atoms. 
Table S1. Results of bond valence sum calculation for TBA $\mathbf{1}$

\begin{tabular}{llllllll} 
Molecule A & & & & & & \\
\hline Ti1A & 4.217 & Ti2A & 4.278 & W3A & 6.294 & W4A & 6.196 \\
W5A & 6.047 & W6A & 5.905 & W7A & 6.263 & W8A & 6.098 \\
W9A & 6.369 & W10A & 6.130 & W11A & 6.072 & W12A & 6.455 \\
Ti1B & 4.351 & Ti2B & 4.535 & W3B & 5.675 & W4B & 5.963 \\
W5B & 6.266 & W6B & 6.173 & W7B & 6.127 & W8B & 5.918 \\
W9B & 6.051 & W10B & 6.365 & W11B & 6.491 & W12B & 6.212 \\
Si1A & 3.859 & Si1B & 3.897 & O101 & 2.126 & O201 & 2.198 \\
O103 & 1.798 & O104 & 1.627 & O105 & 1.750 & O106 & 1.731 \\
O107 & 1.613 & O108 & 1.877 & O109 & 1.703 & O110 & 1.847 \\
O111 & 1.750 & O112 & 1.798 & O203 & 1.529 & O204 & 1.658 \\
O205 & 1.934 & O206 & 1.750 & O207 & 1.658 & O208 & 1.658 \\
O209 & 1.694 & O210 & 2.003 & O211 & 2.080 & O212 & 2.047 \\
O1A & 1.350 & O2A & 1.093 & O3A & 2.187 & O4A & 2.094 \\
O5A & 2.225 & O6A & 2.068 & O7A & 2.014 & O8A & 2.141 \\
O9A & 1.985 & O10A & 2.012 & O11A & 1.993 & O12A & 1.936 \\
O13A & 2.111 & O14A & 1.978 & O15A & 1.979 & O16A & 2.130 \\
O17A & 2.054 & O18A & 1.781 & O19A & 2.055 & O20A & 2.133 \\
O21A & 1.832 & O22A & 2.055 & O23A & 1.860 & O24A & 2.035 \\
O25A & 1.838 & O26A & 1.974 & O27A & 1.902 & O28A & 1.862 \\
O1B & 1.234 & O2B & 1.210 & O3B & 1.911 & O4B & 1.979 \\
O5B & 1.955 & O6B & 1.913 & O7B & 1.847 & O8B & 1.935 \\
O9B & 2.160 & O10B & 1.908 & O11B & 1.822 & O12B & 1.981 \\
O13B & 2.025 & O14B & 1.987 & O15B & 2.072 & O16B & 1.990 \\
O17B & 1.984 & O18B & 1.984 & O19B & 2.055 & O20B & 1.952 \\
O21B & 2.083 & O22B & 2.103 & O23B & 2.018 & O24B & 2.094 \\
\hline & 1.831 & O26B & 1.953 & O27B & 1.921 & O28B & 1.813 \\
\hline
\end{tabular}


Table S1. (continued)

\begin{tabular}{llllllll} 
Molecule B & & & & & & & \\
\hline Ti1C & 4.164 & Ti2C & 3.432 & W3C & 5.940 & W4C & 6.112 \\
W5C & 5.993 & W6C & 6.077 & W7C & 5.979 & W8C & 6.264 \\
W9C & 6.302 & W10C & 5.773 & W11C & 6.170 & W12C & 5.773 \\
Ti1D & 4.067 & Ti2D & 4.243 & W3D & 6.034 & W4D & 6.490 \\
W5D & 5.991 & W6D & 6.260 & W7D & 6.147 & W8D & 6.121 \\
W9D & 6.195 & W10D & 6.033 & W11D & 6.280 & W12D & 5.848 \\
Si1C & 3.868 & Si1D & 3.715 & O301 & 2.123 & O401 & 1.921 \\
O303 & 1.529 & O304 & 1.622 & O305 & 2.003 & O306 & 1.798 \\
O307 & 1.613 & O308 & 1.847 & O309 & 1.898 & O310 & 1.488 \\
O311 & 1.750 & O312 & 1.685 & O403 & 1.658 & O404 & 1.898 \\
O405 & 1.769 & O406 & 2.114 & O407 & 1.566 & O408 & 1.798 \\
O409 & 1.649 & O410 & 1.793 & O411 & 1.971 & O412 & 1.703 \\
O1C & 1.148 & O2C & 1.211 & O3C & 2.148 & O4C & 2.078 \\
O5C & 2.142 & O6C & 2.042 & O7C & 1.974 & O8C & 1.998 \\
O9C & 2.064 & O10C & 2.015 & O11C & 2.062 & O12C & 2.058 \\
O13C & 1.798 & O14C & 1.968 & O15C & 1.962 & O16C & 2.194 \\
O17C & 1.948 & O18C & 1.806 & O19C & 2.068 & O20C & 1.984 \\
O21C & 1.757 & O22C & 2.059 & O23C & 1.856 & O24C & 1.992 \\
O25C & 1.860 & O26C & 1.832 & O27C & 1.931 & O28C & 1.922 \\
O1D & 1.153 & O2D & 1.210 & O3D & 1.807 & O4D & 1.864 \\
O5D & 1.938 & O6D & 1.948 & O7D & 1.924 & O8D & 1.924 \\
O9D & 1.896 & O10D & 1.914 & O11D & 1.950 & O12D & 2.015 \\
O13D & 2.057 & O14D & 2.056 & O15D & 2.078 & O16D & 2.019 \\
O17D & 1.885 & O18D & 2.004 & O19D & 2.018 & O20D & 1.964 \\
O21D & 1.957 & O22D & 2.115 & O23D & 2.095 & O24D & 2.170 \\
\hline
\end{tabular}


Table S2. Results of bond valence sum calculation for CEK $\mathbf{1}$

\begin{tabular}{llllllll}
\hline Ti1 & 4.237 & Ti2 & 4.190 & W3 & 6.063 & W4 & 6.066 \\
W5 & 6.132 & W6 & 6.154 & W7 & 5.985 & W8 & 5.934 \\
W9 & 6.044 & W10 & 6.121 & W11 & 6.151 & W12 & 6.173 \\
Si1 & 3.889 & O101 & 2.077 & O103 & 1.740 & O104 & 1.731 \\
O105 & 1.788 & O106 & 1.803 & O107 & 1.676 & O108 & 1.698 \\
O109 & 1.745 & O110 & 1.736 & O111 & 1.689 & O112 & 1.754 \\
O1 & 1.303 & O2 & 1.184 & O3 & 1.946 & O4 & 1.970 \\
O5 & 1.949 & O6 & 1.964 & O7 & 2.003 & O8 & 1.985 \\
O9 & 1.992 & O10 & 1.939 & O11 & 1.960 & O12 & 1.952 \\
O13 & 2.003 & O14 & 2.024 & O15 & 2.010 & O16 & 2.035 \\
O17 & 1.988 & O18 & 2.002 & O19 & 1.947 & O20 & 1.980 \\
O21 & 1.955 & O22 & 2.063 & O23 & 2.020 & O24 & 2.072 \\
O25 & 1.824 & O26 & 1.840 & O27 & 1.924 & O28 & 1.901 \\
\hline
\end{tabular}

Table S3. Results of bond valence sum calculation for CEK·2

\begin{tabular}{llllllll}
\hline Ti1 & 4.221 & W1 & 6.382 & W2 & 6.342 & W3 & 6.290 \\
W4 & 6.231 & W5 & 6.150 & W6 & 6.268 & Si1 & 3.857 \\
Si1 & 3.857 & O101 & 2.072 & O102 & 1.847 & O103 & 1.949 \\
O104 & 1.949 & O105 & 1.798 & O106 & 1.750 & O1 & 2.154 \\
O2 & 2.254 & O3 & 1.961 & O4 & 2.015 & O5 & 1.985 \\
O6 & 1.987 & O7 & 2.039 & O8 & 2.038 & O9 & 2.066 \\
O10 & 1.984 & O11 & 2.034 & O12 & 1.931 & O13 & 2.038 \\
O14 & 2.152 & O15 & 1.831 & O16 & 1.940 & O17 & 1.933 \\
\hline
\end{tabular}

